\title{
Article \\ Optimization and Performance Assessment of a Logic Selectivity Solution Based on LoRa Communication
}

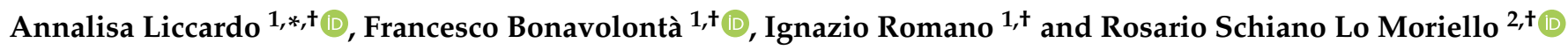 \\ 1 Department of Electrical Engineering and Information Technology, University of Naples Federico II, \\ 80125 Naples, Italy; francesco.bonavolonta@unina.it (F.B.); ignazio.romano@unina.it (I.R.) \\ 2 Department of Industrial Engineering, University of Naples Federico II, 80125 Naples, Italy; \\ rschiano@unina.it \\ * Correspondence: annalisa.liccardo@unina.it; Tel.: +39-081-768-3912 \\ + These authors contributed equally to this work.
}

Citation: Liccardo, A.; Bonavolontà,

F.; Romano, I.; Schiano Lo Moriello, R. Optimization and Performance Assessment of a Logic Selectivity Solution Based on LoRa Communication. Energies 2021, 14, 7359. https://doi.org/10.3390/ en14217359

Academic Editor: Manuel Moreno

Received: 2 October 2021

Accepted: 1 November 2021

Published: 5 November 2021

Publisher's Note: MDPI stays neutral with regard to jurisdictional claims in published maps and institutional affiliations.

Copyright: (c) 2021 by the authors. Licensee MDPI, Basel, Switzerland. This article is an open access article distributed under the terms and conditions of the Creative Commons Attribution (CC BY) license (https:// creativecommons.org/licenses/by/ $4.0 /)$.

\begin{abstract}
Ensuring service continuity has become a fundamental issue for companies involved in electricity distribution; in particular, isolating the smallest possible portion of the network as a result of faults has long been a primary objective. To this aim, solutions based on logic selectivity have been defined and implemented for an efficient search for the network branch affected by the fault and its subsequent isolation. The authors have recently presented a proposal for the implementation of logic selectivity that exploits the LoRa transmission protocol, an ideal solution in the case of areas not reachable by the currently exploited communication technologies. The present paper, instead, deals with the optimization of some LoRa parameters, which made it possible to exploit network configurations in terms of coverage range, sensitivity and signal-to-noise ratio. The performance of the new configuration has been assessed through a number of tests conducted in the laboratory and on-field, highlighting promising results in terms of both intervention times and reliability. In particular, tests conducted in both rural and urban areas have assured fault isolation times as low as $33 \mathrm{~ms}$ (fully compliant with the current regulations) in the presence of the most challenging fault condition.
\end{abstract}

Keywords: electrical distribution network; fault isolation; logic selectivity; LoRa communication; performance assessment; on-field tests

\section{Introduction}

Logic selectivity is a recent approach for the protection of Medium Voltage (MV) networks. According to this approach, the network is equipped with intelligent protection devices capable of locating and isolating a fault simply by exchanging messages with one another [1-3]. This method, compared with the traditional approaches of localization of the faults, guarantees three advantages: (1) the fault is localized in a time that is independent from its position; (2) the number of consumers supplied by the network experiencing a short interruption (less than one minute) during the fault localization is minimized [4,5]; (3) the section of the network that remains powered off to isolate the fault is minimized [6]. In order to make these benefits real and not damage the network as a result of the failure, the rate at which the devices communicate must be adequate [7,8]. As an example, the Italian electricity distributor has planned to install the optical fiber along its MV lines. Since the installation times are high and, in some areas such as the rural ones, uneconomical, the authors in [9-11] proposed a logic selectivity system based on LoRa technology, which has the ease of installation of the wireless networks, but considerable coverage and reliability [12].

Although the technology was promising, in [9], it was noted that in some protocol configurations, communication times led to fault isolation times that were not compliant with the limits tolerated by the regulations. 
Therefore, the authors report hereinafter the results of a research activity aimed at optimizing the protocol, i.e., at the detection of a configuration that, on the one hand, would allow for meeting the time limits of fault isolation, and, on the other hand, maximizes the transmission reliability.

The identified optimal configuration was then assessed through both laboratory and on-field tests by installing the protection devices in real MV/LV (medium voltage to low voltage) substations located on a test line. It was possible to verify the functioning of the system, taking into account real characteristics, such as sources of interference, presence of buildings, realistic distances.

This article is organized as follows: after a brief discussion about the current state of the art methods enabling technologies for logic selectivity implementation in Section 2, the timing characteristics of the protocol are shown and the solution for optimization is illustrated in Section 3. Results obtained in laboratory tests are presented in Section 4. The designed set up for field tests and the related obtained results are described in Section 5. The conclusions are finally reported in Section 6.

\section{Related Works}

Logic selectivity approaches impose the respect of hard time restrictions for communications. This leads to specific issues related to the design of an adequate network infrastructure capable of ensuring fast and reliable communications between electrical devices that can be located even a few kilometers away from one another. Several enabling technologies [13], either wired or wireless, have been considered from both academic and industrial worlds. As for wired solutions, most of the primary substations of the distribution network are already connected in wired wide local area networks (thanks to optic fibers), thus assuring messages transmission with latency values as low as $1 \mathrm{~ms}[6,14]$. Unfortunately, assuring the same performance for distribution lines beyond the primary stations turns out to not always be affordable due to the installation costs of the optic fiber infrastructure. Some projects involving overhead power lines tried to face the problem by installing the fiber below the guard wire; however, possible failure of the wire will surely lead to a break of the optic fiber and, consequently, loss of communication. As can be expected, other wired solutions, for example, Ethernet cables, share similar pros and cons in terms of high performance and installation problems [15].

Thanks to the offered opportunity of transmitting messages on the same cables carrying the electricity, Power Line Communication (PLC) represents a viable solution to reduce installation costs. However, two main limitations affect its exploitation in the logic selectivity applications: limited communication ranges (usually lower than $400 \mathrm{~m}$ ) [16] and the need for installing bypass devices to assure electric continuity in the presence of transformers and switches [17]. Furthermore, the successful operation of this solution can be compromised if the network topology changes after the installation, as in modern smart grids [18].

To suitably overcome the main drawback associated with wired communication networks, solutions exploiting wireless communication technologies, operating in either licensed or free frequency bands [19] can be investigated. As for licensed bands, the communication infrastructure is owned by telecommunications companies, and the related installation and maintenance costs do not directly burden the power companies. As an example, if a mobile network is adopted to realize the connection among electrical substations, power companies have to activate proper subscriptions and buy the associated wireless devices in order to leverage the offered communication services. Subscription costs are mainly associated with the volume of messages to be exchanged and the exploited technology (GSM, 3G, 4G, 5G, or NarrowBand-IoT).

The main drawback associated with this solution relates to the availability and quality of the connection services that directly depend on the specific network provider; as an example, while urban signal coverage can be considered suitable for the proposed application, several rural areas are not supplied, thus preventing the exploitation of such 
solutions [20]. Moreover, the value of latency in message transmission results is comparable with the required intervention time (except for $5 \mathrm{G}$ technology), thus making this technology unfeasible for logic selectivity implementation.

On the other side, free communication frequency bands (the so-called Industrial, Scientific and Medical (ISM) band) can be exploited to deploy a personal wireless communication network among the power substations. As an example, extended WiFi is part of Wireless Local Area Network (WLAN) technologies operating in the ISM bands. The communication range is limited to a few hundred meters (in open space), and the typical power consumption of WiFi devices requires a main power supply, so backup power must be provided in case of failure. The results obtained by adopting this solution for the exchange of messages for logic selectivity implementation are presented in [21] but limited to two devices placed at only $75 \mathrm{~m}$ from one another.

Similar considerations hold for other wireless technologies (as an example, Bluetooth, ZigBee and similar) operating within the ISM band, characterized by nominal coverage ranges of a few hundred meters in open space [22,23]. Moreover, some solutions require handshake protocols that result in transmission overhead, causing undesirable latency in messages exchanging [24].

Finally, other wireless solutions, such as SigFox and LoRaWAN, have been defined in ISM bands to implement wide area networks (range greater than $1 \mathrm{~km}$ ) characterized by reduced power consumption and data rate, thus making them a key aspect in the deployment of low-cost applications for the internet of things. However, the considered solutions suffer from issues of latency and data packet dimension preventing their exploitation for the considered application [25].

To overcome the considered limitations, the authors presented [9] a prototype implementation of a logic selectivity protection of MV distribution networks based on the LoRa communication. Differently from LoRaWAN solutions, the exploited Lora network leverages the reduced packet dimension since no overhead associated with MAC and application layers are present; it is also possible to reduce the message dimension and make its air duration compliant with the time constraint of the logic selectivity. Moreover, the point-to-point connection between successive network nodes further reduces the need to address issues since a node can only communicate with upstream and downstream nodes on carriers characterized by different frequency values [9].

For the sake of the clarity, Table 1 summarizes the compliance of the considered network solutions with the different requirements of the considered application.

Table 1. Comparison of technologies that can be exploited for logic selectivity implementation. Green cell: complaint with logic selectivity implementation. Red cell: not complaint with logic selectivity implementation. Yellow cell: possibly not complaint with logic selectivity implementation (e.g., area not covered by mobile services).

\begin{tabular}{lllll}
\hline Technology & Latency & Installation Costs & Range & Transmission Time \\
\hline Optic fiber & & & \\
\hline Ethernet & & & \\
\hline GSM, 3G, $4 \mathrm{G}$ & & \\
\hline $5 \mathrm{G}$ & & \\
\hline Bluetooth & & \\
Zigbee & & & \\
\hline SigFox & & & \\
\hline LoRaWAN & & \\
\hline LoRa & & \\
\hline
\end{tabular}

\section{LoRa Transmission Optimization}

To better appreciate the optimization brought to the logic selectivity solution, the structure of a LoRa packet as well as its configuration in the previous version are recalled in the following and shown in Figure 1 [26]. The preamble is a sequence of bits required for receiver synchronization. The length of the preamble is programmable but if this is set 
to zero, the transmitter will still transmit two up-chirps, followed by two and a quarter down-chirps [27]. If $T_{\text {sym }}$ indicates the duration of a symbol transmission, the time required to transmit the preamble $T_{\text {preamble }}$ is:

$$
T_{\text {preamble }}=\left(n_{\text {preamble }}+4.25\right) \cdot T_{\text {sym }}
$$

where $n_{\text {preamble }}$ is the length of the preamble in symbols.

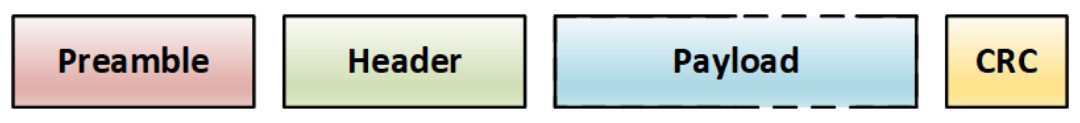

Figure 1. Structure of a LoRa packet.

The header contains essential information to decode the packet, i.e., (1) length of the payload, (2) the value of the Coding Rate (CR) and the presence of the Cyclic Redundancy Check (CRC); it is optional and can be declared explicit or implicit, i.e., present or not in the transmitted packet.

The payload is the content of the message and its length is expressed in symbols. The length in bits of a symbol is a fundamental communication parameter called the Spreading Factor (SF), which can take an integer value between 7 and 12 . The higher the $\mathrm{SF}$, the more robust the communication, since a lower Signal-to-Noise Ratio (SNR) must be guaranteed [28]. On the other hand, the higher the SF, the longer the symbol and the greater the time required for its transmission $[26,29]$. The time required for the transmission of a symbol, $T_{\text {sym }}$ is dependent on SF according to the formula:

$$
T_{\text {sym }}=\frac{2^{\mathrm{SF}}}{\mathrm{BW}}
$$

where BW is the channel bandwidth.

The CR can take integer values from one to four and indicates how many Forward Error Check (FEC) bits are transmitted for every four bits of actual data. Again, a high CR value increases reliability but also increases packet transmission times [30].

For the implementation of logic selectivity, in [9], the preamble length has been set equal to six symbols, since preliminary tests have shown that a shorter preamble does not guarantee synchronization with the reliability needed in this application; the payload length has been set equal to four symbols. Table 2 shows the packet transmission times as a function of SF and BW, obtained using the Lora Calculator [31].

Table 2. Packet transmission time in $\mathrm{ms}$, in dependence of $\mathrm{BW}$ and $\mathrm{SF}$, with $\mathrm{CR}=1$. Values represented in green are compliant with the logic selectivity implementation, while red values are not. Yellow values are very close to the considered time threshold, and the corresponding LoRa configurations

\begin{tabular}{|c|c|c|c|c|c|c|}
\hline BW $\quad$ SF & 7 & 8 & 9 & 10 & 11 & 12 \\
\hline $125 \mathrm{kHz}$ & 30.98 & 61.95 & 123.9 & 206.85 & 413.7 & 827.39 \\
\hline $250 \mathrm{kHz}$ & 15.49 & 30.98 & 61.95 & 103.42 & 206.85 & 413.7 \\
\hline $500 \mathrm{kHz}$ & 7.74 & 15.49 & 30.98 & 51.71 & 103.42 & 206.85 \\
\hline
\end{tabular}
turn out to be unreliable for the implementation.

In order to understand which configurations are suitable for a logic selectivity system, at least theoretically, it is necessary to compare the packet transmission time with a threshold $T_{\text {lim }}$. If a device senses the fault, it waits for a blocking message, called "blind", from downstream device before tripping its circuit. If no message arrives within $T_{\text {lim }}$, the device realizes it is closest to the fault and commands the breaker to trip. The time $T_{\text {lim }}$ is obtained by subtracting from the maximum time allowed for the fault clearing $\left(T_{f_{c}}\right)$ the detection time $T_{d}$ needed by the sensor to detect the fault current and the trip time $T_{t r}$ required by 
the breaker to trip the circuit.

$$
T_{\text {lim }}=T_{f c}-T_{d}-T_{t r}
$$

Referring to the most critical scenario, i.e., a poly-phase short circuit, the maximum admitted time for the fault clearing is $120 \mathrm{~ms}$; typical values of $T_{d}$ and $T_{t r}$ are $27 \mathrm{~ms}$ and $60 \mathrm{~ms}$, respectively. Therefore, Equation (3) gives $T_{\text {lim }}=33 \mathrm{~ms}$.

In Table 2 the SF and BW combinations that do not allow implementation of the logic selectivity are shown in red. In yellow, authors indicate the configurations for which the packet transmission time is less than $T_{\text {lim }}$, but these configurations are discarded because the guard interval is too narrow (it must be always considered that the times in the table are theoretical). Finally, the configurations for which the LoRa communication satisfies the imposed time limits are indicated in green.

In [9], authors carried out various laboratory experiments, which confirmed that the configurations highlighted in green assure to implement a logic selectivity system that meet the time constraints imposed by the regulations.

However, making the configuration reported in yellow available for the network implementation would result in further improvements for the transmission quality, granting superior performance in terms of robustness against noise or interference. In Table 3, in fact, both the SNR that must be guaranteed and the sensitivity $S$ required by the receiver are shown, depending on SF and BW.

Table 3. SNR and S, in dependence on BW and SF.

\begin{tabular}{lccccccc}
\hline BW & SF & $\mathbf{7}$ & $\mathbf{8}$ & $\mathbf{9}$ & $\mathbf{1 0}$ & $\mathbf{1 1}$ & $\mathbf{1 2}$ \\
\hline \multirow{2}{*}{$125 \mathrm{kHz}$} & -7.5 & -10 & -12.5 & -15 & -17.5 & -20 & SNR [dB] \\
& -123 & -126 & -129 & -132 & -134.5 & -137 & S [dBm] \\
\hline \multirow{2}{*}{$250 \mathrm{kHz}$} & -7.5 & -10 & -12.5 & -15 & -17.5 & -20 & SNR [dB] \\
& -120 & -123 & -126 & -129 & -131.5 & -134 & S [dBm] \\
\hline \multirow{2}{*}{$500 \mathrm{kHz}$} & -7.5 & -10 & -12.5 & -15 & -17.5 & -20 & SNR [dB] \\
& -117 & -120 & -123 & -126 & -128.5 & -131 & S [dBm] \\
\hline
\end{tabular}

Therefore, a fundamental step of the research activity relied on the optimization of the packet, in order to obtain configurations of the protocol characterized by higher coverage, sensitivity, and reliability.

If the devices involved in the communication already know the communication parameters, the header may not be transmitted. Then, the first step was to declare the implicit header. This requires that (1) the communication parameters are set in advance and all devices in the network know them; (2) the parameters remain constant, at least until all devices are reprogrammed.

The second operation consisted in disabling the CRC. The CRC is used by the receiver to verify the integrity of the received data and, if necessary, to request the re-transmission of the message. It should be considered that, as a safety mechanism, if a device detects a fault and does not receive the blocking message within $T_{\text {lim }}$, it commands the tripping of the circuit. Thus, if a device receives a corrupted message, there is no time to ask the receiver to re-transmit the packet before the breaker must be tripped.

With the optimized packet, the transmission times provided by the LoRa Calculator are shown in Table 4.

Table 4. Transmission time in ms of the optimized packet, in dependence of BW and SF.

\begin{tabular}{|c|c|c|c|c|c|c|}
\hline $\begin{array}{ll}\text { BW } & \text { SF }\end{array}$ & 7 & 8 & 9 & 10 & 11 & 12 \\
\hline $125 \mathrm{kHz}$ & 25.86 & 51.71 & 103.42 & 165.89 & 331.78 & 663.55 \\
\hline $250 \mathrm{kHz}$ & 12.93 & 25.86 & 51.71 & 82.94 & 165.89 & 331.78 \\
\hline $500 \mathrm{kHz}$ & 6.46 & 12.93 & 25.86 & 41.47 & 82.94 & 165.89 \\
\hline
\end{tabular}


It can be seen that other configurations (SF,BW), which considerably improve the reliability of communication, such as (7125), (8250) and (9500) can also be taken into account.

However, the times shown in Table 4 are theoretical times. In order to assess the realized system, the communication between the devices has been tested through both laboratory and field tests.

\section{Assessment with Laboratory Tests}

The laboratory tests were conducted on the demonstrator shown in Figure 2. It consists of a radial MV network, represented by the copper conductors to which the consumers are connected. Several switches are arranged along the line, controlled by the intelligent devices. In particular, $\mathrm{CP}$ indicates the protection in the primary substation of the line, ICSx (with $x=1,2,3$ ) represents the protection device in the secondary substations; a line with three secondary substations has been assumed. The device indicated with $\mathrm{BCB}$ represents the Boundary Circuit Breaker, i.e., the switch that is closed to re-power the line downstream of the fault through a primary backup cabin.

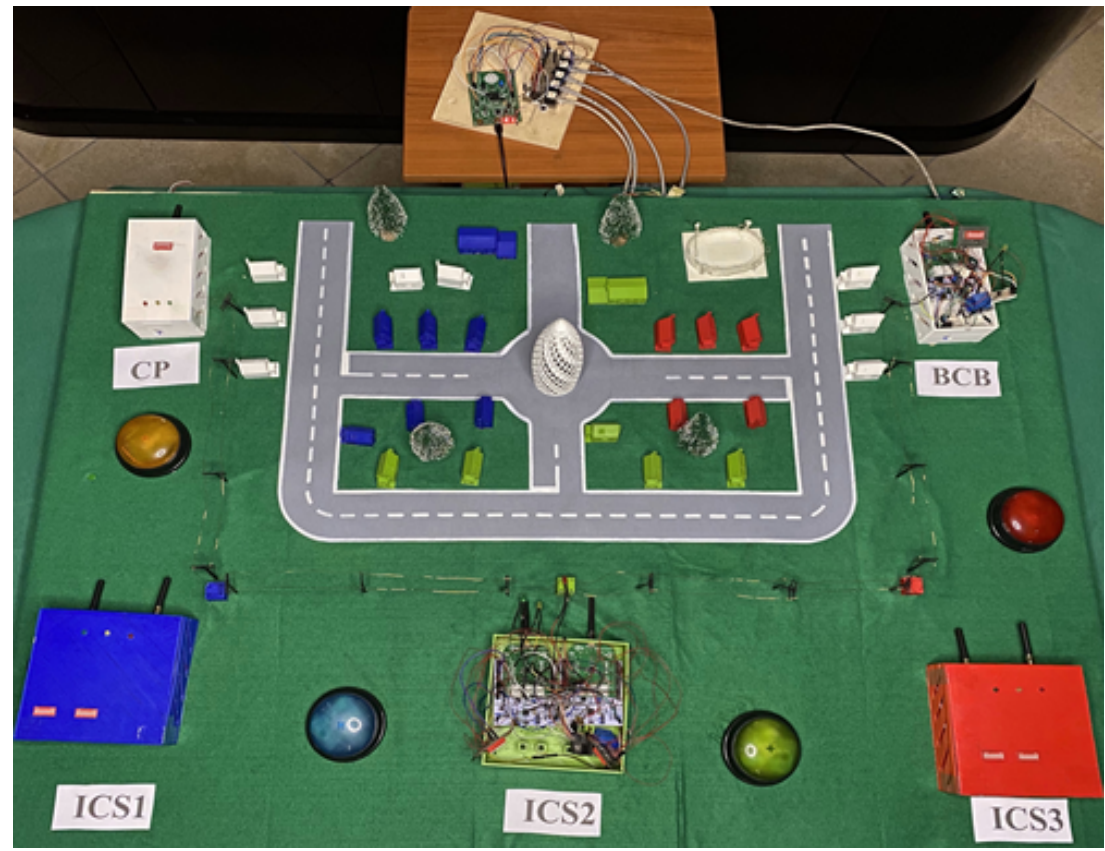

Figure 2. Demonstrator developed for laboratory tests.

Except for $\mathrm{CP}$ and $\mathrm{BCB}$, each device contains two communication boards: the first one has to transmit messages to the upstream device, the second one has to receive messages from the downstream device. The communication boards (observable in the open box of ICS2 and BCB) have been realized by adopting the expansion board I-NUCLEOSX1272D [32] by STMicroelectronics, which includes transceiver LoRa Semtech SX1272. The expansion board is connected to a board NUCLEO-L073RZ [33] by STMicroelectronics, based on the 32-bit, ultra-low-power, STM32L0 ARM Cortex microcontroller.

All the devices are connected to a controller board that has two tasks: (1) to send the fault signal to the devices, emulating, for each of them, the output of the fault current sensor; (2) to acquire the digital signal of message reception and switch closure of all the devices, in order to perform the time interval measurements.

Each device is equipped with an eight-position DIP switch, through which it is possible to set a combination of bits that are read at start-up by the boards and, according to them, $\mathrm{BW}, \mathrm{SF}, \mathrm{CR}$, presence of CRC and header of the communication are set, without the need to reprogram the devices. This feature is particularly useful when installing the device, 
as it may not be straightforward to connect the programmer to all devices to change the communication parameters.

A button is placed between each substation, which, when pressed, simulates the fault condition at that point. This feature is useful for one-shot tests, mainly used for demonstrating purposes; when repeated tests have to be carried out, instead, the controller is adopted to generate the fault signals.

For each set SF and BW configuration, four test conditions were considered, placing the fault at four different locations. For a better understanding of the measured intervals, a fault occurring between ICS2 and ICS3 is considered; the corresponding application of the logic selectivity approach for fault isolation is shown in Figure 3. When a fault occurs in a radial distribution line (Figure $3 a$ ), each substation experiencing the high current sends a blind message to the upstream substation (Figure $3 \mathrm{~b}$ ) in order to prevent the tripping of its circuit breaker. If a substation measuring the fault current does not receive the blind message within a defined time interval, it trips its own circuit breaker and makes the downstream substation perform the same operation by sending an appropriate message (Figure 3c). Finally, a message is forwarded downstream the distribution line towards the so-called tie-closure to allow the power supply of the last portion of the network from another parallel distribution line (Figure 3d). This way, the fault is isolated and the smaller portion of line and associated consumers is dropped from the power distribution.

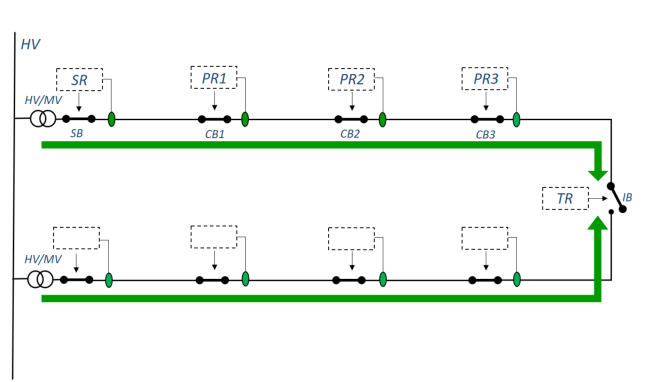

(a)

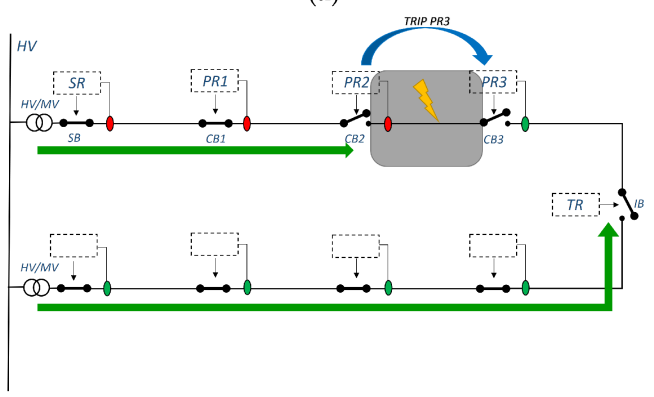

(c)

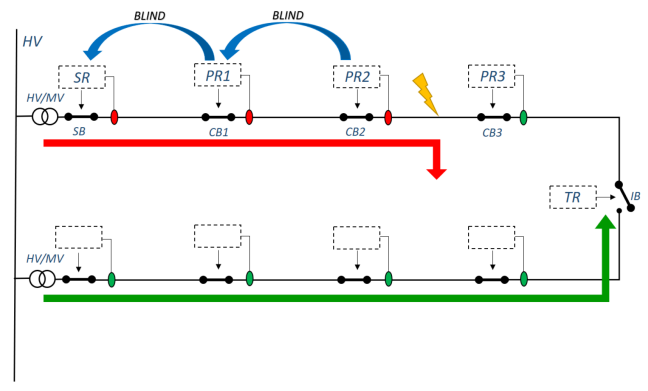

(b)

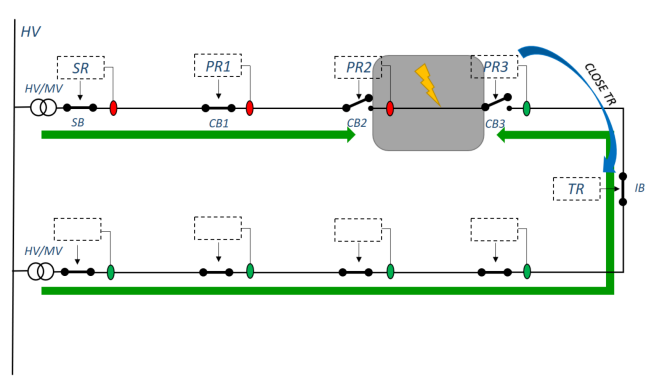

(d)

Figure 3. Example of logic selectivity application for faults occurring between ICS2 and ICS3. (a) Healthy conditions. (b) Fault between protection relay 2 (PR2) and PR3; transmission of blind signals. (c) Circuit breaker 2 (CB2) tripping, and transmission of the Trip signal to PR3. (d) CB3 tripping and transmission of the Close signal to Tie recloser (TR). [9].

As for the implemented prototype, Figure 4 shows the digital signals acquired by the controller board. For each device, the line CB is associated with the circuit breaker position (high logical level corresponds to the close circuit condition, while low logical level identifies the open circuit condition); Rx line is an interrupted line from the Lora expansion board whose edge rises each time a message is received. 


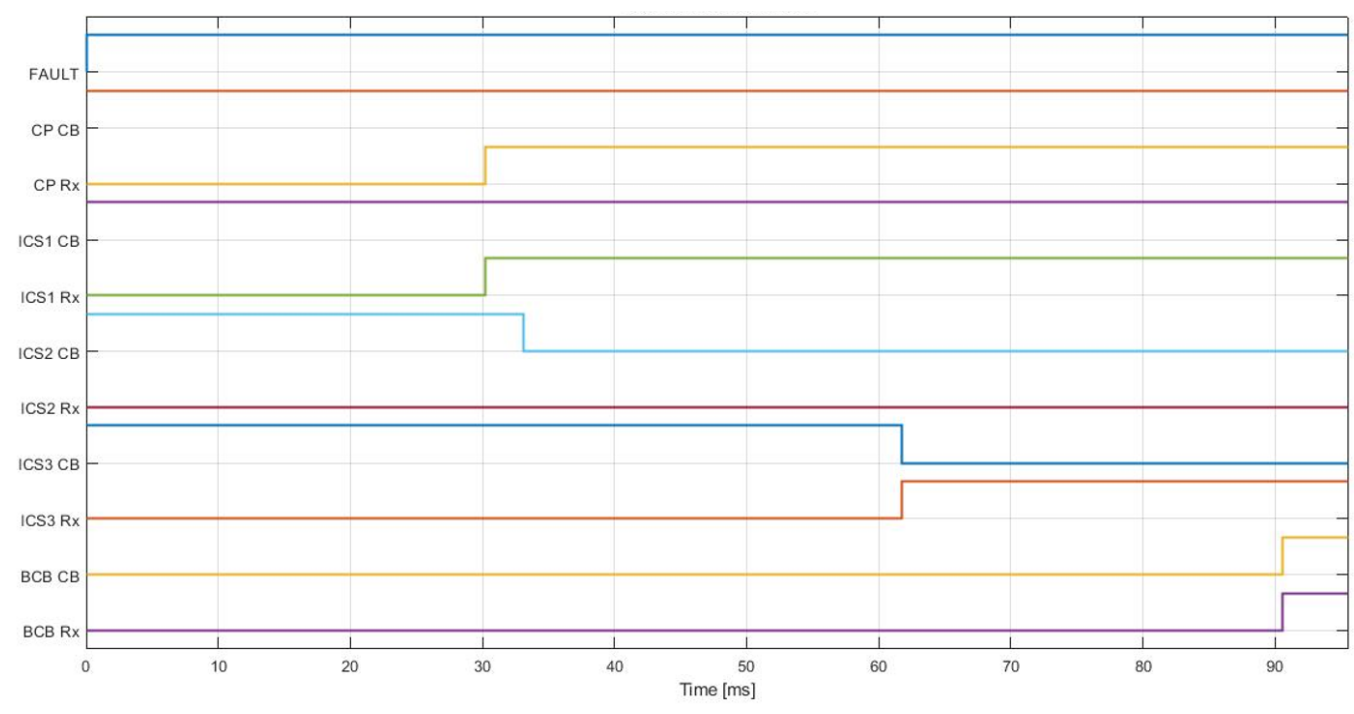

Figure 4. Digital signals acquired during a fault between ICS2 and ICS3.

The time in which the fault signal is generated (blue signal at the top) is taken as the zero reference instant. The device in the primary substation senses the fault current, but receives the "blind" signal from the device ICS1 before the time $T_{\text {lim }}$ (equal to $33 \mathrm{~ms}$ ) expires; in fact, the $\mathrm{CP} \mathrm{Rx}$ line exhibits a rising edge, which indicates the reception of the message. Therefore, the device in CB keeps the switch closed (the CP CB switch line has no transitions). The device in ICS1 also senses the fault and receives the "blind" signal from the downstream device within $T_{\text {lim }}$ (rising edge of ICS1 Rx line) and keeps the substation switch closed. The device in ICS2 senses the fault, but does not receive the "blind" signal in time (because ICS3, that is located downstream of the fault, does not detect it) and, after the time $T_{\text {lim }}$ has elapsed, commands the tripping of the circuit breaker (falling edge of line ICS2 CB).

In order to re-power the consumers downstream of the fault, the ICS2 device, after having tripped the circuit, sends an opening command to the device immediately downstream, i.e., ICS3; this can be observed from the rising edge of the ICS3 Rx line and the ICS3 CB line. At this point the section of line affected by the fault has been isolated and it is possible to re-power the consumers downstream of ICS3. To this end, the ICS3, after opening, transmits the closure message to the downstream device to be forwarded to the BCB. The BCB device is immediately downstream of ICS3, so, after about $90 \mathrm{~ms}$, the rising edge associated with the closing signal of $B C B C B$ can be observed. If the fault occurs between ICS1 and ICS2, for example, the closure message for the BCB is transmitted from ICS2 to ICS3; the latter forwards the message to the $\mathrm{BCB}$, with an obvious increase in time. In any case, the $\mathrm{BCB}$ closure operation is less time critical than the fault isolation, since the maximum time allowed for the re-powering of the consumers downstream of the fault is $1 \mathrm{~s}$ [34].

In order to assess the efficacy of the optimized transmission, different tests have been carried out in the laboratory with the implemented network prototype to measure the fault isolation times; in particular, tests have been carried out for each of the three new configurations (i.e., SF 7 and BW $125 \mathrm{kHz}, \mathrm{SF} 8$ and BW $250 \mathrm{kHz}$, SF 9 and BW $500 \mathrm{kHz}$ ) and for four different positions of the fault along the distribution line.

In order to assess the communication, for each scenario the following times were measured:

- The time at which a device received the "blind" signal (indicated in the tables in green);

- The time at which a device, not having received the "blind" signal, opened the circuit (indicated in the tables in red);

- The time at which a device received the "open" signal (indicated in the tables in blue); 
- The time at which a device received the "close" signal for the BCB (indicated in the tables in magenta).

It is worth noting that the time measured on each device strictly depends on the specific fault applied to the prototype network. As an example, for fault occurring between ICS1 and ICS2, the measured time corresponds to the tripping of the breaker, while it corresponds to the reception of a blind message for the fault occurring between ICS2-ICS3. It has to be noted that if a fault occurs between ICS3 and BCB, the "close" signal to BCB is not transmitted (NT), as there are no sections to be re-powered downstream of the fault.

For each of the test conditions (configuration and fault position), 100 repeated trials were performed and the results will be presented in terms of average measured time and associated confidence intervals obtained with a coverage factor equal to three. Moreover, the reliability has been assessed for each test condition, by counting the number of trials in which all devices have correctly received messages in time and operated according to the logic selectivity approach.

The corresponding measurement results are shown in Tables 5-7 for LoRa configurations equal to respectively to SF 7 and BW $125 \mathrm{kHz}$, SF 8 and BW $250 \mathrm{kHz}$ and SF 9 and BW $500 \mathrm{kHz}$.

Table 5. Results obtained with $\mathrm{SF}=7$ and $\mathrm{BW}=125 \mathrm{kHz}$.

\begin{tabular}{lcccc}
\hline Fault Location & CP-ICS1 & ICS1-ICS2 & ICS2-ICS3 & ICS3-BCB \\
\hline$T_{C P}[\mathrm{~ms}]$ & $33.092 \pm 0.003$ & $30.177 \pm 0.003$ & $30.180 \pm 0.003$ & $30.179 \pm 0.003$ \\
$T_{I C S 1}[\mathrm{~ms}]$ & $67.88 \pm 0.13$ & $32.975 \pm 0.003$ & $30.187 \pm 0.003$ & $30.185 \pm 0.003$ \\
$T_{I C S 2}[\mathrm{~ms}]$ & $96.65 \pm 0.13$ & $61.625 \pm 0.003$ & $33.085 \pm 0.003$ & $30.163 \pm 0.003$ \\
$T_{I C S 3}[\mathrm{~ms}]$ & $125.97 \pm 0.13$ & $90.572 \pm 0.003$ & $61.730 \pm 0.003$ & $32.922 \pm 0.003$ \\
$T_{B C B}[\mathrm{~ms}]$ & $155.27 \pm 0.13$ & $119.872 \pm 0.003$ & $90.572 \pm 0.003$ & $\mathrm{NT}$ \\
Reliability & $100 \%$ & $100 \%$ & $99 \%$ & $99 \%$ \\
\hline
\end{tabular}

Table 6. Results obtained with SF $=8$ and $\mathrm{BW}=250 \mathrm{kHz}$.

\begin{tabular}{lcccc}
\hline Fault Location & CP-ICS1 & ICS1-ICS2 & ICS2-ICS3 & ICS3-BCB \\
\hline$T_{C P}[\mathrm{~ms}]$ & $33.084 \pm 0.003$ & $30.171 \pm 0.003$ & $30.168 \pm 0.003$ & $30.170 \pm 0.003$ \\
$T_{I C S 1}[\mathrm{~ms}]$ & $67.83 \pm 0.12$ & $32.983 \pm 0.003$ & $30.178 \pm 0.003$ & $30.177 \pm 0.003$ \\
$T_{I C S 2}[\mathrm{~ms}]$ & $96.60 \pm 0.12$ & $61.628 \pm 0.003$ & $33.081 \pm 0.003$ & $30.161 \pm 0.003$ \\
$T_{I C S 3}[\mathrm{~ms}]$ & $125.92 \pm 0.12$ & $90.562 \pm 0.003$ & $61.721 \pm 0.003$ & $32.921 \pm 0.003$ \\
$T_{B C B}[\mathrm{~ms}]$ & $155.22 \pm 0.12$ & $119.862 \pm 0.003$ & $90.564 \pm 0.003$ & $\mathrm{NT}$ \\
Reliability & $100 \%$ & $100 \%$ & $100 \%$ & $99 \%$ \\
\hline
\end{tabular}

Table 7. Results obtained with $\mathrm{SF}=9$ and $\mathrm{BW}=500 \mathrm{kHz}$.

\begin{tabular}{lcccc}
\hline Fault Location & CP-ICS1 & ICS1-ICS2 & ICS2-ICS3 & ICS3-BCB \\
\hline$T_{C P}[\mathrm{~ms}]$ & $33.078 \pm 0.003$ & $30.211 \pm 0.003$ & $30.213 \pm 0.003$ & $30.213 \pm 0.003$ \\
$T_{I C S 1}[\mathrm{~ms}]$ & $67.71 \pm 0.12$ & $32.971 \pm 0.003$ & $30.212 \pm 0.003$ & $30.213 \pm 0.003$ \\
$T_{I C S 2}[\mathrm{~ms}]$ & $96.48 \pm 0.12$ & $61.650 \pm 0.003$ & $33.088 \pm 0.003$ & $30.198 \pm 0.003$ \\
$T_{I C S 3}[\mathrm{~ms}]$ & $125.91 \pm 0.12$ & $90.600 \pm 0.003$ & $61.762 \pm 0.003$ & $32.923 \pm 0.003$ \\
$T_{B C B}[\mathrm{~ms}]$ & $155.21 \pm 0.12$ & $119.900 \pm 0.003$ & $90.602 \pm 0.003$ & $\mathrm{NT}$ \\
Reliability & $100 \%$ & $98 \%$ & $100 \%$ & $99 \%$ \\
\hline
\end{tabular}

The measured times are compliant with the logic selectivity approach and very close to the theoretical values given by the LoRa calculator. It can be seen that the "blind" signals are received in time less than $T_{l i m}$ with a probability never lower than $99 \%$. It can be deduced that the packet optimization allows implementation of a logic selectivity system with LoRa protocol configurations characterized by greater reliability and robustness. 


\section{Assessment with Field Tests}

\subsection{Developed Hardware and Software}

In order to carry out the field tests, the LoRa protection devices were installed at distances of hundreds of meters. It is therefore not possible to exploit wired connections between the protection devices and the controller to transmit the fault signal simultaneously to all the devices and to acquire the digital signals for time measurements, as previously done in the laboratory tests.

At this aim, authors designed a control node to be connected to each LoRa protection device, suitable for field testing and having the same functions as the laboratory controller. The components of the node are shown separately in Figure 5.

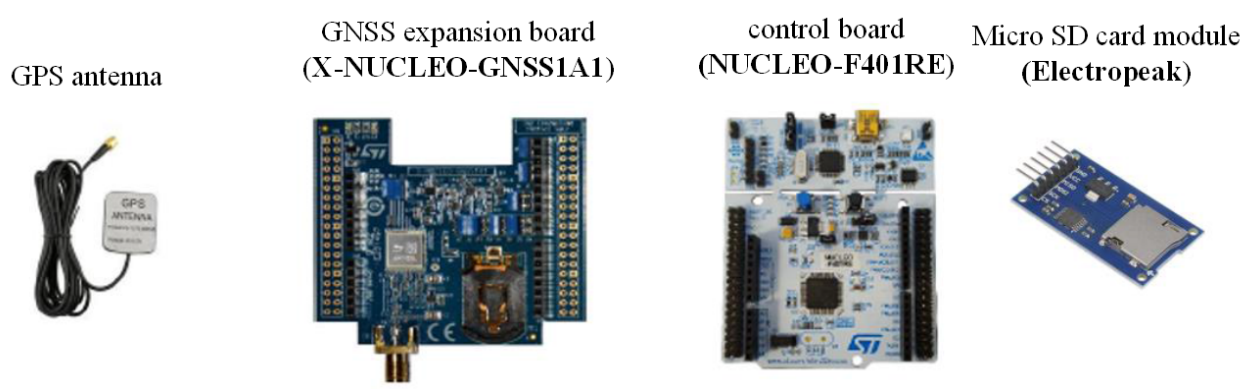

Figure 5. Components adopted for the control node in field tests.

The main part of the node consists of a board NUCLEO-F401RE [35] by STMicroelectronics, based on a 32-bit ARM CORTEX-M4 microprocessor with a maximum CPU frequency of $84 \mathrm{MHz}[36,37]$. An X-NUCLEO-GNSS1A1 expansion board with GPS (Global Positioning System) antenna [38], based on the Teseo-LIV3F GNSS (Global navigation satellite system) module, featuring a $26 \mathrm{MHz}$ temperature compensated oscillator crystal and a dedicated $32 \mathrm{kHz}$ RTC (Real Time Clock), is used to send a fault signal synchronized with the other devices. An Electropeak Micro SD Card Module was used to record the measured times.

The connections between the NUCLEO-F401RE and the other components of the node are shown in Figure 6. The board first distributes the power and ground level to all other components. It is connected to the GNSS with the Serial Receive and Transmit pins, to communicate with the module through UART (Universal Asynchronous Receiver/Transmitter) protocol. In addition, the board also receives the Pulse Per Second (PPS) signal from the GNSS. Communication with the Micro SD Card module takes place via Serial Peripheral Interface (SPI) protocol; therefore, the CS (Chip Select), MISO (Master Input Slave Output), MOSI (Master Output Slave Input) and SCL (Serial Clock) lines are connected.

Finally, the board is connected to the LoRa protection device through three lines: (1) LoRa Rx: the edges of this line signal that the protection device has received a message ("blind", "open" or "BCB close"); (2) CB: this is the digital line through which the protection device controls the circuit breaker; its edges signal the commanded tripping or closing of the breaker; (3) fault: digital line, controlled by the NUCLEO-F401RE, whose edges signal to the LoRa device the presence of a fault; (4) reset: digital line that returns the protection device to its initial state in order to perform a new trial. In order to create a unique control node suitable for all the protection devices in the network, the NUCLEO-F401RE always manages all five fault lines (the one to be connected to the CP device, to ICS1, ICS2, ICS3 and $\mathrm{BCB}$ ); depending on the type of device that is installed, it will only be connected to one of these lines. 


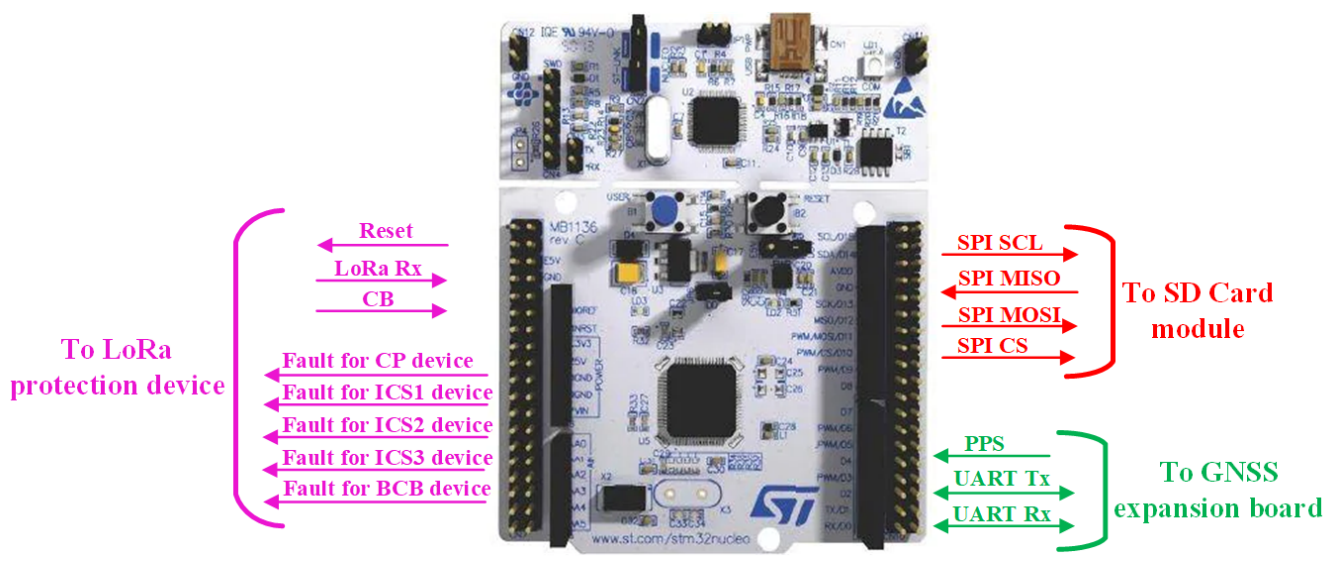

Figure 6. Connection between the control board and the other components of the node.

Figure 7 shows the protection device with the controller node assembled.

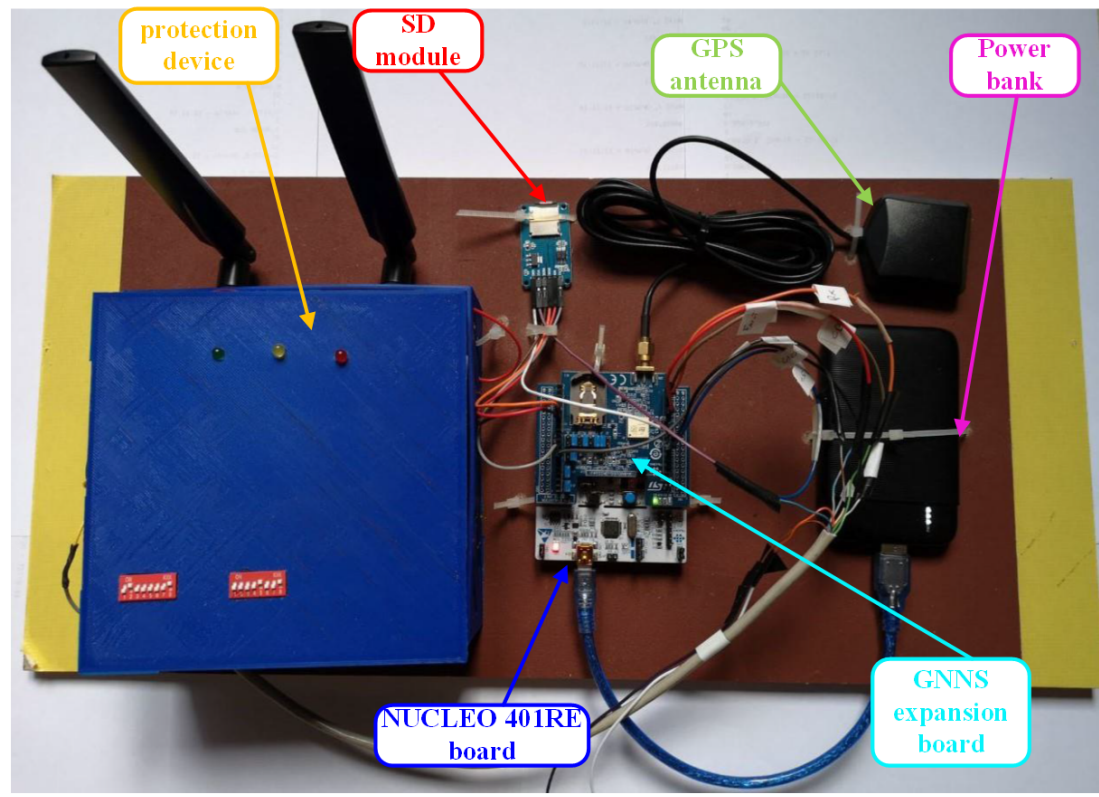

Figure 7. Protection device and control node assembled for field tests.

As for the firmware of the NUCLEO-F401RE board, it has been developed in the Mbed environment, in order to perform the following operations:

1. Configuration of the input and output lines, initialization of the UART and SPI communications and creation of the file in the SD card.

2. Communication with GNSS is established and the string containing position, date and time provided by the GPS is isolated; according to NMEA standard [39], it is sufficient to isolate the string with "\$GPRMC" header; position and time are saved in the SD card file. In particular, the second value of the current date is retained to provide the successive trigger event for the tests execution.

3. The internal time is updated in an interrupt routine triggered by the rising edge of the PPS signal; tests carried out by ST Microelectronics show that $100 \%$ of the Teseo-LIV3F PPS pulses deviate from $1 \mathrm{~s}$ by $12.5 \mathrm{~ns}$; therefore, in order to generate the fault simultaneously on all the devices, it was preferred to use the PPS for time update, rather than an internal timer on the core board that could present some drift problems.

4. Every five seconds a fault is generated, following the step sequence: rising edge of the reset signal, waiting $1 \mathrm{~s}$, setting of the lines for fault generation, waiting for a 
maximum of $2 \mathrm{~s}$ of the Rx reception signals or CB switch command, measurement and saving on SD card of the time intervals between the fault and the Rx and CB edges.

As for the LoRa parameters, the configuration involving SF and BW equalling, respectively, 8 and $250 \mathrm{kHz}$, has been adopted; moreover, a transmission power of $10 \mathrm{dBm}$ has been set. In order to test different fault positions simultaneously on all the protection devices, the status of the lines for fault generation is changed every $5 \mathrm{~s}$ and is set in dependence on the updated seconds value, according to the values shown in Table 8 .

Table 8. Values of fault lines to test different fault positions. The values in red indicate the lines for which a fault signal is generated; on the contrary, green values highlights not-faulty portion of the distribution line.

\begin{tabular}{ccccccc}
\hline Second Values & $\begin{array}{c}\text { Fault } \\
\text { CP }\end{array}$ & $\begin{array}{c}\text { Fault } \\
\text { ICS1 }\end{array}$ & $\begin{array}{c}\text { Fault } \\
\text { ICS2 }\end{array}$ & $\begin{array}{c}\text { Fault } \\
\text { ICS3 }\end{array}$ & $\begin{array}{c}\text { Fault } \\
\text { BCB }\end{array}$ & $\begin{array}{c}\text { Fault } \\
\text { Position }\end{array}$ \\
\hline $0,5,10$ & 1 & 0 & 0 & 0 & 0 & CP-ICS1 \\
\hline $15,20,25$ & 1 & 1 & 0 & 0 & 0 & ICS1-ICS2 \\
\hline $30,35,40$ & 1 & 1 & 1 & 0 & 0 & ICS2-ICS3 \\
\hline $45,50,55$ & 1 & 1 & 1 & 1 & 0 & ICS3-BCB \\
\hline
\end{tabular}

In one minute, all fault positions are tested three times, so 180 trials of all the fault positions are assessed within $60 \mathrm{~min}$.

\subsection{Obtained Results}

The tests were carried out in two different scenarios, one in a rural area with few obstacles in the line of sight and another in an urban area with buildings, interference and other obstacles [40]. In both cases the optimized packet, with SF equal to 8 and BW equal to $250 \mathrm{kHz}$ have been selected.

In the rural area, Figure 8 shows the map of the area where the tests were carried out with the respective position of the various nodes. The LoRa nodes were positioned along the road at a distance of about $300 \mathrm{~m}$ from one another; the node positions for these tests have been chosen in order to represent as much as possible a real scenario. In fact, the nodes have been installed near real secondary substations.

In Table 9 the results obtained on the time measurements in 180 trials for each fault position are presented.

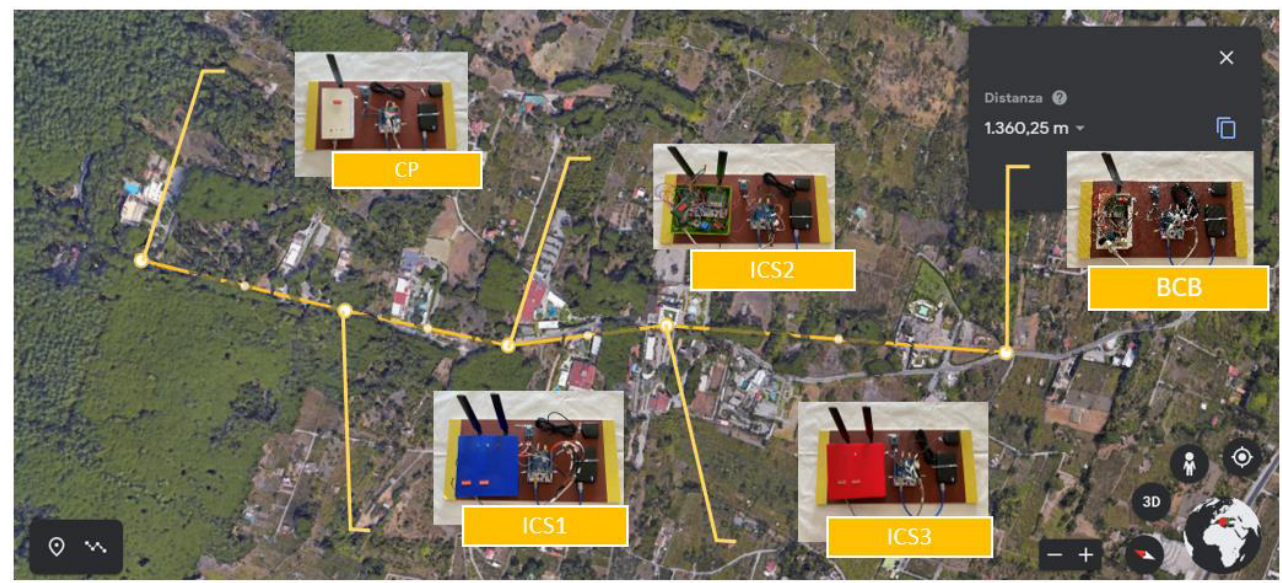

Figure 8. Position of devices for tests in rural environments.

Also in this case, the confidence intervals obtained from the repeated time measurements, with a coverage factor $k$ equal to three have been evaluated. 
Table 9. Results obtained in rural areas.

\begin{tabular}{lcccc}
\hline Fault Location & CP-ICS1 & ICS1-ICS2 & ICS2-ICS3 & ICS3-BCB \\
\hline$T_{C P}[\mathrm{~ms}]$ & $32.986 \pm 0.003$ & $30.063 \pm 0.006$ & $30.064 \pm 0.006$ & $30.068 \pm 0.006$ \\
$T_{I C S 1}[\mathrm{~ms}]$ & $68.514 \pm 0.003$ & $32.989 \pm 0.003$ & $30.094 \pm 0.003$ & $30.096 \pm 0.003$ \\
$T_{I C S 2}[\mathrm{~ms}]$ & $97.287 \pm 0.003$ & $61.633 \pm 0.003$ & $32.974 \pm 0.006$ & $30.070 \pm 0.003$ \\
$T_{I C S 3}[\mathrm{~ms}]$ & $126.608 \pm 0.003$ & $90.478 \pm 0.003$ & $61.714 \pm 0.006$ & $32.916 \pm 0.006$ \\
$T_{B C B}[\mathrm{~ms}]$ & $155.816 \pm 0.12$ & $119.70 \pm 0.09$ & $90.45 \pm 0.05$ & $\mathrm{NT}$ \\
Reliability & $97.8 \%$ & $98.3 \%$ & $98.9 \%$ & $98.3 \%$ \\
\hline
\end{tabular}

It can be noted that the "blind" messages arrive in time to prevent the unnecessary tripping of circuit breakers upstream of the fault (about $30 \mathrm{~ms}$ in all the fault positions). Moreover, the fault is isolated before the time limit of $33 \mathrm{~ms}$ has elapsed. The re-powering of the network after the fault also takes place in good time, since, in any case, the $\mathrm{BCB}$ is closed in much less time than $1 \mathrm{~s}$. All the measured times are compatible with those measured in laboratory. The results are also satisfactory in terms of reliability, which is always greater than $97 \%$.

In order to demonstrate that it is possible to implement logic selectivity with LoRa technology even in areas where there are obstacles and interference, other tests have been carried out positioning the LoRa nodes in an urban area, at a distance of about $100 \mathrm{~m}$ from one another, with buildings that obstructed the line of sight. The position of nodes is shown in Figure 9.

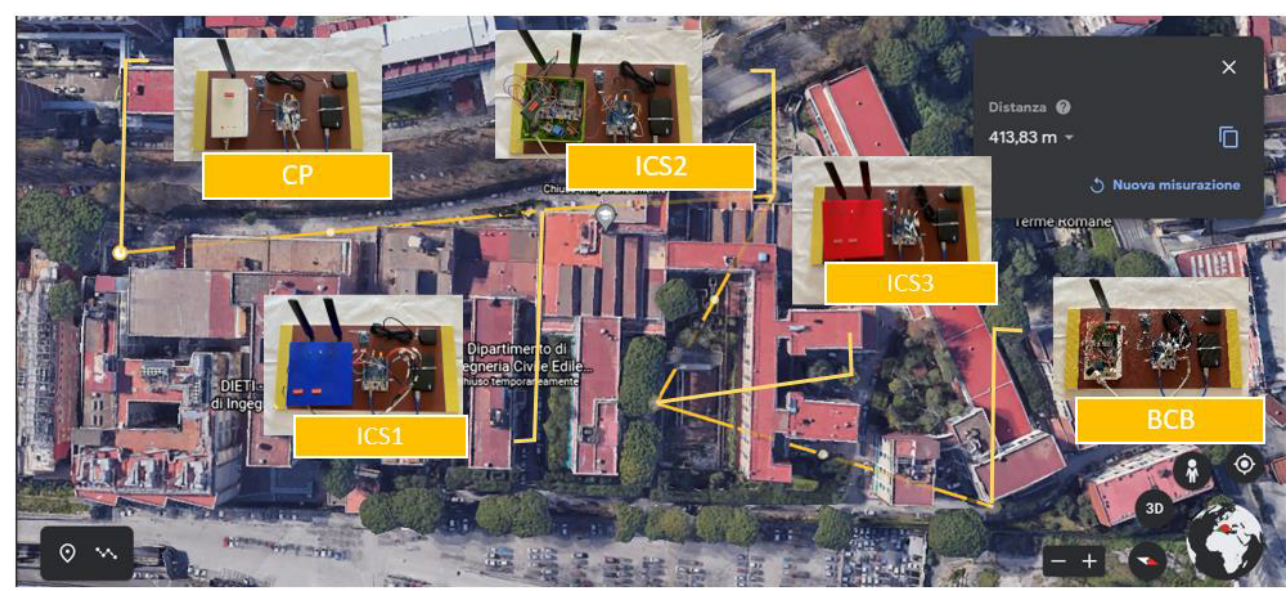

Figure 9. Position of devices for tests in urban environment.

The obtained results are reported in Table 10.

Table 10. Results obtained in urban areas.

\begin{tabular}{lcccc}
\hline Fault Location & CP-ICS1 & ICS1-ICS2 & ICS2-ICS3 & ICS3-BCB \\
\hline$T_{C P}[\mathrm{~ms}]$ & $32.986 \pm 0.003$ & $30.056 \pm 0.003$ & $30.056 \pm 0.003$ & $30.057 \pm 0.003$ \\
$T_{I C S 1}[\mathrm{~ms}]$ & $68.490 \pm 0.003$ & $32.987 \pm 0.003$ & $30.067 \pm 0.003$ & $30.086 \pm 0.006$ \\
$T_{I C S 2}[\mathrm{~ms}]$ & $97.268 \pm 0.003$ & $61.628 \pm 0.003$ & $32.960 \pm 0.006$ & $30.065 \pm 0.006$ \\
$T_{I C S 3}[\mathrm{~ms}]$ & $126.588 \pm 0.003$ & $90.456 \pm 0.003$ & $61.701 \pm 0.006$ & $32.919 \pm 0.003$ \\
$T_{B C B}[\mathrm{~ms}]$ & $155.888 \pm 0.003$ & $119.759 \pm 0.003$ & $90.458 \pm 0.003$ & $\mathrm{NT}$ \\
Reliability & $97.2 \%$ & $98.9 \%$ & $98.3 \%$ & $98.3 \%$ \\
\hline
\end{tabular}

Results similar to those observed in tests in rural areas can be appreciated. It is worth noting that the reliability has values close to those estimated in the rural environment. This is because the effect of the obstacles is compensated by the lower distance between the devices. 


\section{Conclusions}

The problem of an efficient implementation of the logic selectivity approach for the protection of MV distribution networks has been considered and faced in the paper. Limitations affecting typical wired and wireless solutions (i.e., installation costs, coverage range and latency) have been overcome by means of a proposal of wireless networks based on LoRa technology. In particular, the attention has been focused on the optimization of the transmission parameters (SF, BW, header e CRC) of the LoRa packet in such a way as to simultaneously improve coverage range, SNR and reliability.

The proposed solution has been assessed in a number of tests conducted both in laboratory and on-field experiments. As for the lab tests, they were mandated to verify the correct functioning of the realized network prototype; for all the considered conditions of LoRa parameter values and fault position in the emulated distribution line, the prototype has been capable of clearing the fault and isolating only the faulty branch within times compliant with the current regulations (below $33 \mathrm{~ms}$ for the worst fault condition). Moreover, the solution has shown a high reliability, with a success rate never lower than $99 \%$ for all test conditions.

To carry out tests in actual operating configurations, the problem of synchronizing the fault event on the network nodes far away from one another has to be faced. A new node has been designed and implemented for the purpose. In particular, a GNSS module has been exploited to provide the trigger event for the fault occurrence, while a further embedded system has been allowed to control the wireless node lines and measure the required times. Tests have been conducted in both rural and urban environments, highlighting a remarkable reliability thanks to success rates greater than $97 \%$.

Ongoing activities are mainly focused on the assessment of a security layer to protect the network from undesired hack attacks. The layer has been defined and designed by considering:

- The reduced number of messages that the nodes exchange (due to the reduced number of faults that affect the distribution line); the possibility to determine the sequence is reduced because of the reduced number of samples.

- The reduced intervention time $(30 \mathrm{~ms})$ that makes man-in-the-middle type attacks ineffective; the eventual attacker has little time to apply brute force attacks to determine the correct message.

- In the case of an attempted attack, as soon as a node receives a message that does not conform to the security layer being used and recognizes the danger, it deactivates and releases control to a time-selectivity approach, safeguarding the critical electrical infrastructure.

Author Contributions: Conceptualization, A.L., F.B. and R.S.L.M.; data curation, R.S.L.M. and I.R.; investigation, R.S.L.M. and I.R.; methodology, A.L. and R.S.L.M.; software, F.B. and I.R.; supervision, A.L., F.B. and R.S.L.M.; writing—original draft, I.R.; writing—review \& editing, A.L. All authors have read and agreed to the published version of the manuscript.

Funding: This research received no external funding.

Institutional Review Board Statement: Not applicable.

Informed Consent Statement: Not applicable.

Data Availability Statement: Data sharing is not applicable to this article.

Acknowledgments: The authors would like to thank A. Smith, M. D'Angelo and S. Cannavacciuolo from STMicroelectronics at Arzano (Italy) for both the offered opportunity of testing the proposed solution on STM32 microcontrollers and the technical support during the execution of the experimental tests.

Conflicts of Interest: The authors declare no conflict of interest. 


List of Abbreviations
LoRa
MV
LV
PLC
GSM
ISM
WLAN
MAC
CR
CRC
SF
BW
SNR
FEC
S
CP
ICSx
BCB
GPS
GNSS
RTC
UART
PPS
SPI
CS
MISO
MOSI
SCL
NMEA
List of math symbols
$T_{\text {sym }}$
$T_{\text {preamble }}$
$n_{\text {preamble }}$
$T_{\text {lim }}$
$T_{f c}$
$T_{d}$
$T_{t r}$
$T_{C P}$
$T_{I C S x}$
$T_{B C B}$

Long Range

Medium Voltage

Low Voltage

Power Line Communication

Global System for Mobile Communications

Industrial, Scientific and Medical

Wireless Local Area Network

Medium Access Control

Coding Rate

Cyclic Redundancy Check

Spreading Factor

Bandwidth

Signal-to-noise ratio

Forward Error Check

Sensitivity

Primary substation

$x$-th Secundary Substation

Boundary Circuit Breaker

Global Positioning System

Global Navigation Satellite System

Real Time Clock

Universal Asynchronous Receiver/Transmitter

Pulse per Second

Serial Peripheral Interface

Chip Select

Master Input Slave Output

Master Output Slave Input

Serial Clock

National Marine Electronics Association

LoRa symbol transmission time

LoRa preamble transmission time

Number of symbols included in LoRa preamble

Maximum allowed time for blind message exchange

Maximum allowed time for blind fault clearing

Time required for fault detection

Time required for circuit tripping

Measured time for operations of the Primary Substation

Measured time for operations of the $x$-th Secundary Substation

Measured time for operations of the Boundary Circuit Breaker

\section{References}

1. $\quad$ Prévé, C. Protection of Electrical Networks; John Wiley \& Sons: Hoboken, NJ, USA, 2013.

2. IEC 61850: Communication Networks and Systems in Substations; Standard; International Electrotechnical Commission: Geneva, Switzerland, 2013.

3. Nam, S.R.; Ko, W.H.; Key, S.; Kang, S.H.; Lee, N.H. IEC 61850-Based Centralized Protection against Single Line-To-Ground Faults in Ungrounded Distribution Systems. Energies 2021, 14, 722. [CrossRef]

4. Sterle, C.; Caragallo, V.; Liccardo, A.; Masone, A.; Fatica, A.; Bonavolonta, F. Integer Linear Programming for optimizing the position of IEDs in Medium Voltage smart grids. In Proceedings of the 2021 IEEE International Instrumentation and Measurement Technology Conference (I2MTC), Glasgow, UK, 17-20 May 2021; [CrossRef]

5. Dede, A.; Della Giustina, D.; Franzoni, F.; Pegoiani, A. IEC 61850-based logic selectivity scheme for the MV distribution network. In Proceedings of the 2014 IEEE International Workshop on Applied Measurements for Power Systems Proceedings (AMPS), Aachen, Germany, 24-26 September 2014; pp. 1-5. [CrossRef] 
6. Della Giustina, D.; Ferrari, P.; Flammini, A.; Rinaldi, S.; Sisinni, E. Automation of distribution grids with IEC 61850: A first approach using broadband power line communication. IEEE Trans. Instrum. Meas. 2013, 62, 2372-2383. [CrossRef]

7. Cepeda, C.; Orozco-Henao, C.; Percybrooks, W.; Pulgarín-Rivera, J.D.; Montoya, O.D.; Gil-González, W.; Vélez, J.C. Intelligent fault detection system for microgrids. Energies 2020, 13, 1223. [CrossRef]

8. Heidari, S.; Fotuhi-Firuzabad, M.; Lehtonen, M. Planning to equip the power distribution networks with automation system. IEEE Trans. Power Syst. 2017, 32, 3451-3460. [CrossRef]

9. Angrisani, L.; Bonavolontà, F.; Liccardo, A.; Schiano Lo Moriello, R. On the Use of LoRa Technology for Logic Selectivity in MV Distribution Networks. Energies 2018, 11, 3079. [CrossRef]

10. Bonavolontà, F.; Caragallo, V.; Fatica, A.; Liccardo, A.; Masone, A.; Sterle, C. Optimization of IEDs Position in MV Smart Grids through Integer Linear Programming. Energies 2021, 14, 3346. [CrossRef]

11. Bonavolontà, F.; Caputi, C.; Liccardo, A.; Teotino, A. Protection of MV smart grid based on IoT technology. In Proceedings of the 2019 II Workshop on Metrology for Industry 4.0 and IoT (MetroInd4.0 IoT), Naples, Italy, 4-6 June 2019; pp. 112-116. [CrossRef]

12. Rizzi, M.; Ferrari, P.; Flammini, A.; Sisinni, E. Evaluation of the IoT LoRaWAN Solution for Distributed Measurement Applications IEEE Trans. Instrum. Meas. 2017, 66, 3340-3349. [CrossRef]

13. Bonavolontà, F.; Tedesco, A.; Moriello, R.S.L.; Tufano, A. Enabling wireless technologies for industry 4.0: State of the art. In Proceedings of the 2017 IEEE International Workshop on Measurement and Networking (M\&N), Naples, Italy, 27-29 September 2017; pp. 1-5. [CrossRef]

14. Della Giustina, D.; Dedè, A.; Invernizzi, G.; Valle, D.P.; Franzoni, F.; Pegoiani, A.; Cremaschini, L. Smart Grid Automation Based on IEC 61850: An Experimental Characterization. IEEE Trans. Instrum. Meas. 2015, 64, 2055-2063. [CrossRef]

15. Sauter, T.; Lobashov, M. End-to-End Communication Architecture for Smart Grids. IEEE Trans. Ind. Electron. 2011, 58, 1218-1228. [CrossRef]

16. Della Giustina, D.; Andersson, L.; Iglesias, G.J.R. Experimental performance characterization of a meshed network for the smart grid. In Proceedings of the Innovative Smart Grid Technologies Europe (ISGT EUROPE), 2013 4th IEEE/PES, Lyngby, Denmark, 6-9 October 2013; pp. 1-5. [CrossRef]

17. Cataliotti, A.; Cosentino, V.; Cara, D.D.; Tine, G. Simulation and Laboratory Experimental Tests of a Line to Shield Medium-Voltage Power-Line Communication System. IEEE Trans. Power Deliv. 2011, 26, 2829-2836. [CrossRef]

18. Chandna, V.K.; Zahida, M. Effect of Varying Topologies on the Performance of Broadband Over Power Line. IEEE Trans. Power Deliv. 2010, 25, 2371-2375. [CrossRef]

19. Angrisani, L.; Bonavolontà, F.; Tocchi, A.; Moriello, R.S.L. Frequency domain measurement node based on compressive sampling for sensors networks. In Proceedings of the 2015 IEEE International Workshop on Measurements \& Networking (M\&N), Coimbra, Portugal, 12-13 October 2015; pp. 1-5. [CrossRef]

20. Review of Mobile Communication and the 5G in Manufacturing. Procedia Manuf. 2019, 32, 600-612. [CrossRef]

21. Parikh, P.P.; Sidhu, T.S.; Shami, A. A comprehensive investigation of wireless LAN for IEC 61850-based smart distribution substation applications. IEEE Trans. Ind. Inform. 2013, 9, 1466-1476. [CrossRef]

22. Sales Mendes, A.; Jiménez-Bravo, D.M.; Navarro-Cáceres, M.; Reis Quietinho Leithardt, V.; Villarrubia González, G. Multi-Agent Approach Using LoRaWAN Devices: An Airport Case Study. Electronics 2020, 9, 1430. [CrossRef]

23. Mateos Matilla, D.; Murciego, Á.L.; Jiménez-Bravo, D.M.; Sales Mendes, A.; Leithardt, V.R. Low-cost Edge Computing devices and novel user interfaces for monitoring pivot irrigation systems based on Internet of Things and LoRaWAN technologies. Biosyst. Eng. 2021. [CrossRef]

24. Cho, K.; Park, W.; Hong, M.; Park, G.; Cho, W.; Seo, J.; Han, K. Analysis of Latency Performance of Bluetooth Low Energy (BLE) Networks. Sensors 2015, 15, 59-78. [CrossRef]

25. Mekki, K.; Bajic, E.; Chaxel, F.; Meyer, F. A comparative study of LPWAN technologies for large-scale IoT deployment. ICT Express 2019, 5, 1-7. [CrossRef]

26. Sornin, N.; Luis, M.; Eirich, T.; Kramp, T.; Hersent, O. Lorawan Specification; LoRa Alliance: Grenoble, France, 2015.

27. Reynders, B.; Pollin, S. Chirp spread spectrum as a modulation technique for long range communication. In Proceedings of the 2016 Symposium on Communications and Vehicular Technologies (SCVT), Mons, Belgium, 22 November 2016; pp. 1-5. [CrossRef]

28. Paredes-Parra, J.M.; Garcìa-Sànchez, A.J.; Mateo-Aroca, A.; Molina-Garcìa, A. An Alternative Internet-of-Things Solution Based on LoRa for PV Power Plants: Data Monitoring and Management. Energies 2019, 12, 881. [CrossRef]

29. Fang, H.; Tan, R.; Han, Y.; Chen, X.; Zhao, J. An experimental analysis of SNR performance for LoRa communication. In Proceedings of the 2018 IEEE 4th International Conference on Computer and Communications (ICCC), Chengdu, China, 7-10 December 2018; pp. 57-64. [CrossRef]

30. Bor, M.; Vidler, J.E.; Roedig, U. LoRa for the Internet of Things. In Proceedings of the EWSN '16 Proceedings of the 2016 International Conference on Embedded Wireless Systems and Networks; Junction Publishing: EWSN 2016, Graz, Austria, 15-17 February 2016; [CrossRef]

31. Semtech. SX1272 LoRa Calculator. Available online: https://semtech.my.salesforce.com/sfc/p/\#E0000000JelG/a/2R00000 0HUhK/6T9Vdb3_ldnElA8drIbPYjs1wBbhlWUXej8ZMXtZXOM (accessed on 1 October 2021). 
32. STMicroelectronics. High-Performance RF Transceiver Expansion Board Featuring FSK, OOK and the LoRa Long Range Modem for STM32 Nucleo-64. Available online: https:/ / www.st.com/en/evaluation-tools/i-nucleo-sx1272d.html (accessed on 1 October 2021).

33. STMicroelectronics. STM32 Nucleo-64 Development Board with STM32L073RZ MCU, Supports Arduino and ST Morpho Connectivity. Available online: https://www.st.com/en/evaluation-tools/nucleo-1073rz.html (accessed on 1 October 2021).

34. 568/2019/R/EEL: Update of the Tariff Regulation of Electricity Transmission, Distribution and Metering Services for the Period 2020-2023 (in Italian); Standard; Regulatory Authority for Energy, Networks and Environment (ARERA): Milan, Italy, 2019.

35. STMicroelectronics. STM32 Nucleo-64 Development Board with STM32F401RE MCU, Supports Arduino and ST Morpho Connectivity. Available online: https:/ /www.st.com/en/evaluation-tools/nucleo-f401re.html (accessed on 1 October 2021).

36. Bonavolontà, F.; D'Apuzzo, M.; Liccardo, A.; Vadursi, M. New approach based on compressive sampling for sample rate enhancement in DASs for low-cost sensing nodes. Sensors 2014, 14, 18915-18940. [CrossRef]

37. Capriglione, D.; Carratù, M.; Liguori, C.; Paciello, V.; Sommella, P. A soft stroke sensor for motorcycle rear suspension. Measurement 2017, 106, 46-52. [CrossRef]

38. Carratù, M.; Iacono, S.D.; Pietrosanto, A.; Paciello, V. IMU self-alignment in suspensions control system. In Proceedings of the 2019 IEEE International Instrumentation and Measurement Technology Conference (I2MTC), Auckland, New Zealand, 20-23 May 2019; pp. 1-6. [CrossRef]

39. Proprietary, P. National Marine Electronics Association NMEA 2000 Edition 3.00 Release. Bulletin 2013, 13, 122.

40. Marais, J.M.; Malekian, R.; Abu-Mahfouz, A.M. LoRa and LoRaWAN testbeds: A review. In Proceedings of the 2017 IEEE Africon, Cape Town, South Africa, 18-20 September 2017; pp. 1496-1501. [CrossRef] 